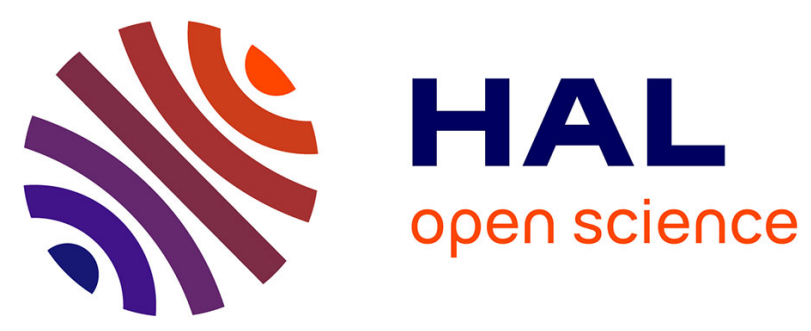

\title{
Three-Dimensional Analytical Surface Quantification of Heterogeneous Silica-Alumina Catalyst Supports
}

Lucian Roiban, Ovidiu Ersen, Charles Hirlimann, Marc Drillon, Alexandra

Chaumonnot, Laurent Lemaitre, Anne-Sophie Gay, Loïc Sorbier

\section{- To cite this version:}

Lucian Roiban, Ovidiu Ersen, Charles Hirlimann, Marc Drillon, Alexandra Chaumonnot, et al.. ThreeDimensional Analytical Surface Quantification of Heterogeneous Silica-Alumina Catalyst Supports. ChemCatChem, 2017, 9 (18), pp.3503 - 3512. 10.1002/cctc.201600543 . hal-01701427

\section{HAL Id: hal-01701427 \\ https://hal-ifp.archives-ouvertes.fr/hal-01701427}

Submitted on 26 Jun 2019

HAL is a multi-disciplinary open access archive for the deposit and dissemination of scientific research documents, whether they are published or not. The documents may come from teaching and research institutions in France or abroad, or from public or private research centers.
L'archive ouverte pluridisciplinaire HAL, est destinée au dépôt et à la diffusion de documents scientifiques de niveau recherche, publiés ou non, émanant des établissements d'enseignement et de recherche français ou étrangers, des laboratoires publics ou privés. 


\title{
Three-Dimensional Analytical Surface Quantification of Heterogeneous Silica-Alumina Catalyst Supports
}

\author{
Lucian Roiban, ${ }^{*[a, b, c]}$ Ovidiu Ersen, ${ }^{*[a]}$ Charles Hirlimann, ${ }^{[a]}$ Marc Drillon, ${ }^{[a]}$ \\ Alexandra Chaumonnot, ${ }^{[b]}$ Laurent Lemaitre, ${ }^{[b]}$ Anne-Sophie Gay, ${ }^{[b]}$ and Loïc Sorbier ${ }^{[b]}$
}

The ability of energy-filtered transmission electron microscopy (EFTEM) tomography to provide 3D chemical maps at the nanoscale opens a new way to analyse heterogeneous materials quantitatively. In association with other techniques, EFTEM tomography has been employed in the study of amorphous silica-alumina catalyst supports. Two types of samples prepared either by mechanical mixing (MM) or by the precipitation of silica on boehmite (PSB) that have similar proportions of silica and alumina were analysed. The sample synthesised by the PSB method shows a smaller degree of heterogeneity than the sample obtained by MM. For both types of samples, a higher concentration of alumina was found at the surface, whereas silica mostly constituted the core of the sample. A thermal treatment in a humid atmosphere was shown to redistribute the silica inside the sample as well as on its surface, which decreased the specific surface area at the same time. The acid sites localisation was defined as a specific curve at the interface between the two components upon reaching the surface of the support. The length of this curve, the "alumina-silica boundary line", was estimated by using EFTEM tomography and discussed qualitatively with the chemical inter-mixing information deduced from additional techniques such as FTIR and NMR spectroscopy.

\section{Introduction}

The chemical industry, in general, and the oil refining industry in particular, customs a large range of catalysts from homogeneous to heterogeneous in various forms such as metal complexes, metals deposited on a support or as oxides. Among them, solid acid catalysts are used widely for their ability to break $\mathrm{C}-\mathrm{C}$ bonds. For this family of materials, several models allow the prediction of their chemical activity and the type of active sites. Amorphous silica-alumina, zeolites, sulfate metal oxides and hetero polyacids, characterised by the different nature of the acid sites and acid strength, are typical examples of such materials. ${ }^{[1]}$

Amorphous silica-alumina mixtures are mesoporous materials characterised by a moderate acidity that are generally less active than zeolites. ${ }^{[2,3]}$ Used in bi-functional reactions, they

[a] Dr. L. Roiban, Prof. O. Ersen, Dr. C. Hirlimann, Dr. M. Drillon Institut de Physique et Chimie des Matériaux de Strasbourg (IPCMS) UMR 7504 CNRS-Université de Strasbourg (UdS)

23, rue du Lœss, 67037 Cedex 08 Strasbourg (France)

E-mail: lucian.roiban@insa-lyon.fr ersen@ipcms.unistra.fr

[b] Dr. L. Roiban, Dr. A. Chaumonnot, L. Lemaitre, Dr. A.-S. Gay, Dr. L. Sorbier IFPEnergies nouvelles, Rond-point de l'échangeur de Solaize BP 3, 69360 Solaize (France)

[c] Dr. L. Roiban

Université de Lyon, INSA-Lyon, MATEIS, SNMS

7, Avenue Jean Capelle, 69621 Villeurbanne Cedex (France) limit uneconomical over-cracking reactions. ${ }^{[4]}$ They are employed preferentially as catalyst supports in hydrocarbon reactions such as the hydrocracking of the Vacuum Gaz Oil fraction (VGO) or Fisher-Tropsch waxes, olefins oligomerisation and dewaxing. ${ }^{[5,6]}$ Anyway, the precise nature of the acid sites provided by the silica-alumina mixture to be used for the mentioned reactions is still under investigation. The main difficulties are related to the lack of accurate characterisation techniques that are selective to the surface chemistry and the $3 \mathrm{D}$ character of the grains. A bi-functional catalyst requires an additional hydrogenating function provided by a sulfide or a metallic active phase deposited on the support surface. The silica-alumina mesoporosity enhances the molecular diffusion and reduces the confinement effects compared to the micropores of zeolites, which provides an essential balance between the acid function of the support and the hydrogenation function of the deposited active phase to avoid the over-cracking process. ${ }^{[7,8]}$ The acidic properties of mesoporous silica-alumina materials depend on the molar ratio of $\mathrm{SiO}_{2}$ and $\mathrm{Al}_{2} \mathrm{O}_{3}$, the degree of homogeneity or heterogeneity and the accessibility to the acid sites. ${ }^{[4]}$ To increase the number of acid sites on the support surface during the synthesis, $\mathrm{Al}$ in tetragonal coordination should be in as large an amount as possible. From a more general point of view, the control of the acidity of amorphous silicaalumina materials requires a precise understanding and optimisation of the synthesis method. ${ }^{[5]}$ Among the different methods used systematically, thermal treatment can play a significant role by changing the texture significantly ${ }^{[9,10]}$ and dispersing the silica or alumina in the support. ${ }^{[4]}$ The difference in behaviour after the steaming of materials produced by the graft- 
ing of molecular silica precursors on alumina and the grafting of molecular alumina precursors on silica gives an insight to the kind of mobile species under steaming conditions. ${ }^{[11,12]}$ Steaming has an effect on the acidic sites on both kinds of samples. However, the texture of steamed $\mathrm{Si} / \mathrm{Al}_{2} \mathrm{O}_{3}$ materials is much less affected than that of steamed $\mathrm{Al} / \mathrm{SiO}_{2}$. Si prevents $\mathrm{Al}_{2} \mathrm{O}_{3}$ sintering, whereas $\mathrm{Al}$ cannot prevent $\mathrm{SiO}_{2}$ sintering. This leads to the conclusion that steaming mainly produces mobile Si species that can deposit a few tens of microns away from their initial position, which was observed in the steaming of a mechanical mixture of $50 \mu \mathrm{m}$ alumina grains and $70 \mu \mathrm{m}$ fluid catalytic cracking beads. ${ }^{[13]}$

Recently, a complex mechanism has been proposed for the formation of acid sites by using DFT calculations. ${ }^{[14]}$ The activity in the hydro-conversion of $n$-heptane is proportional to the number of Brønsted acid sites for a large family of silica-alumina complexes from mesoporous alumina-silicates to ultra-stabilised $Y$ zeolite. ${ }^{[15]}$ After the analysis of a large set of samples obtained by several synthesis methods by using ${ }^{27} \mathrm{Al} N \mathrm{NR}$ spectroscopy, it was concluded that the strength of the acidic sites is governed by the diffusion of Al atoms into the silica network. ${ }^{[16]}$ As catalytic reactions occur at the surface of the support, the quantitative determination of the distribution of silica and alumina at the pore surface is crucial to model the catalytic properties. Several studies have been dedicated to the measurement of the relative amount of $\mathrm{Al}$ and $\mathrm{Si}$ based on various techniques such as X-ray microanalysis ${ }^{[17]}$ in TEM, for instance, but the resulting information gives a relatively poor spatial resolution and suffers from a poor signal-to-noise ratio (SNR). Another technique used currently is ${ }^{27} \mathrm{Al}$ NMR spectroscopy, which can provide information about the coordination of $\mathrm{Al}$ atoms in the volume of the all existent phases. Surface information with regard to the acid sites distribution can be obtained, for instance, by using FTIR spectroscopy by the adsorption of basic molecular probes such as $\mathrm{CO}$, pyridine, ammonia, quinolone or diazines. ${ }^{[18]}$ In this case, CO molecules are generally adsorbed more preferentially on the strong Lewis acid sites than on the medium or soft ones and finally on the strongest to the softest Brønsted sites. ${ }^{[19]}$ The catalytic tests on model molecules such as the quoted $n$-heptane hydro-isomerisation test or the cumene-cracking test ${ }^{[20]}$ provide a good characterisation of the surface acidity. Nevertheless, these approaches only give a mean behaviour with no direct information of the surface composition and homogeneity, which is restricted to the molecular probes or model molecules used. Those Brønsted and Lewis acid sites are obtained by combining amorphous alumina and silica to form aluminosilicates, and their density depends strongly on the synthesis method. ${ }^{[21]}$ Brønsted acid sites are formed by the substitution of a Si atom by tetrahedral $\mathrm{Al}^{[22]}$ and the Lewis acid sites are formed on the surface of $\mathrm{Al}^{3+}$ by removing ${ }^{[23]}$ an $\mathrm{OH}^{-}$.

The structural and chemical characteristics of the aluminosilicates employed in bi-functional catalysis influence the dispersion of the active phase. ${ }^{[24]}$ Consequently, the chemical mapping of the support surface is a step forward in the characterisation of these catalyst supports.
In this context, a combination of the energy-filtered transmission electron microscopy (EFTEM) ${ }^{[25,26]}$ and electron tomography approaches ${ }^{[27,28]}$ can provide a real $3 D$ chemical map $^{[29-31]}$ of a material with a complex morphology, ${ }^{[32,33]}$ which offers new perspectives for the analysis of catalyst supports and catalytic process modelling. EFTEM tomography could, in principle, give valuable information on both the surface chemistry and reactant accessibility for catalysis applications. ${ }^{[34]}$ Moreover, this technique has the advantage to visualise the analysed grains directly, which is often helpful to explain synthesis mechanisms or the microscopic modelling of macroscopic properties.

The aim of this work is to correlate the synthesis of amorphous silica-alumina catalyst supports with their acidic properties and the chemical distribution of silica and alumina at the surface by employing EFTEM tomography quantitatively in association with other more traditional techniques, such as $X$-ray fluorescence (XRF) spectroscopy, X-ray photoelectron spectroscopy (XPS), FTIR spectroscopy and magic-angle-spinning (MAS) NMR spectroscopy. We focused our work on two amorphous silica-alumina materials characterised by single domains of pure silica and alumina synthesised by mechanical mixing (MM) and the precipitation of silica on boehmite (PSB). To obtain the final catalysts, each sample was submitted to a thermal treatment in a humid atmosphere to result in four different materials. The contact at the surface between individual silica and alumina domains leads to the formation of aluminosilicate with catalytic acid properties. The intersection between the surface of the silica-alumina interface and the external surface of the grain is thus fundamental for the acid character of the grain. In this context, the goal of this study was also to estimate the length of this "surface contact line" to be correlated to the chemical information on the inter-mixing of the two components deduced by using more traditional spectroscopic techniques such as NMR and FTIR spectroscopy.

\section{Results}

\section{Bulk and surface analysis}

The bulk analysis performed by using XRF spectroscopy (Table S2) shows that all the samples are composed of $70 \mathrm{wt} \%$ alumina and $30 \mathrm{wt} \%$ silica as targeted initially during the synthesis. Notably, water-treated samples were not analysed as apparently neither Al nor Si loss occurs during this kind of treatment.

Surface analysis provided by XPS (Figure S1) reveals a symmetric peak of Si $2 p$ with a binding energy (BE) between 102 and $103 \mathrm{eV}$, which is close to that of pure silica $(B E=102.3 \mathrm{eV})$. With regard to the Al signal, only sample AS1 exhibits an asymmetric peak that corresponds to Al2p, which can be decomposed into two contributions, one at $\mathrm{BE}=73.9 \mathrm{eV}$ in the range of the pure transitions of alumina (from $B E=73.6 \mathrm{eV}$ for $\alpha$-alumina to $\mathrm{BE}=74.4 \mathrm{eV}$ for $\gamma$-alumina) and the other at $\mathrm{BE}=$ $75.06 \mathrm{eV}$ compatible with an aluminosilicate phase.

The quantification of the XPS spectra (Table S3) shows that alumina is predominant at the surface of the samples. The 
thermal treatment generates an increase of $\mathrm{Si}$ atoms at the surface of the first sample. For the second sample, basically the same ratio between silica and alumina is preserved at the surface.

The measurements performed by using $\mathrm{N}_{2}$ adsorption (Table S4) demonstrate that the specific surface of the samples decreases after the thermal treatment has been performed. The main observation is that the total pore volume remains constant and the pore diameter increases slightly.

"Adsorption-selective" FTIR spectra in the region of the $-\mathrm{OH}$ stretching band can be extracted from the subtraction of the spectrum in the absence of $\mathrm{CO}$ species from the spectrum at high $\mathrm{CO}$ coverage if the Brønsted acid sites are saturated. In the raw FTIR spectra of samples recorded in the region of the $-\mathrm{OH}$ stretching bands before $\mathrm{CO}$ adsorption (Figure S2), the different bands can be attributed following the theoretical results obtained by $\mathrm{DFT}^{[35]}$ based on the hypothesis that the Al$\mathrm{OH}$-type hydroxyl group belongs to the surface of $\gamma$-alumina grains. If we compare the spectra of the untreated and thermally treated samples, the band of basal $\mathrm{OH}-\mu_{1}-\mathrm{Al}^{\mathrm{IV}}$ at around $\tilde{v}=3789 \mathrm{~cm}^{-1}$ disappears and a new band that corresponds to $\mathrm{H}$-bonded $\mathrm{SiOH}$ or basal $\mathrm{OH}-\mu_{2}-\mathrm{Al}^{\mathrm{VI}}$ near $\tilde{\mathrm{v}}=3689 \mathrm{~cm}^{-1}$ appears. The spectral modification is more pronounced for AS1 than for AS2. We observe that the band that corresponds to $\mathrm{Si}-\mathrm{Al}-\mathrm{OH}-$ type hydroxyl groups is hidden completely in the very wide band of $\mathrm{Si}-\mathrm{OH}$-type hydroxyl groups. Finally, after the extraction procedure described here, except for AS1, a band around $\tilde{v}=3450 \mathrm{~cm}^{-1}$ was observed for all the samples (Figure S3), which is considered to be characteristic of strong Brønsted acid sites. ${ }^{[21]}$

The extracted FTIR spectra recorded in the region that corresponds to the $\mathrm{C}-\mathrm{O}$ stretch can provide quantitative data with regard to the type of acid sites by recording consecutive spectra during the successive $\mathrm{CO}$ pulses (Figure S4). It is known that the $\mathrm{CO}$ molecules are first adsorbed by the strongest Lewis acid sites at low coverage. Therefore, the absorption band around $\tilde{v}=2230 \mathrm{~cm}^{-1}$ is attributed to the interaction of the strong Lewis acid sites and that around $\tilde{v}=2200 \mathrm{~cm}^{-1}$ is caused by the soft Lewis acid sites (Figure S4) as described previously. ${ }^{[36,37]}$ The interaction between $\mathrm{CO}$ and the $\mathrm{Al}-\mathrm{OH}-$ type hydroxyl group can be observed in the absorption band recorded at $\tilde{v}=2160 \mathrm{~cm}^{-1}$ and that with the Si-OH-type hydroxyl group is at $\tilde{v}=2140 \mathrm{~cm}^{-1}$. The band recorded at $\tilde{v}=$ $2170 \mathrm{~cm}^{-1}$ is attributed to strong Brønsted acid sites, the atomic structure of which is still under debate. ${ }^{[36-40]}$ Their strength distribution is revealed by the shift of the band with the fractional coverage. No band at $\tilde{v}=2170 \mathrm{~cm}^{-1}$ is detected at a lower $\mathrm{CO}$ coverage (Figure $\mathrm{S} 4$ ) and at $\mathrm{CO}$ saturation (Figure S5) in the spectra of AS1, which indicates that no strong Brønsted acid sites are observed on this sample by FTIR spectroscopy, as already observed from traditional FTIR spectroscopy. With regard to the AS1-Water sample, the recorded signal shows that the thermal treatment creates Brønsted acid sites at the surface of the sample. The band at $\tilde{v}=2170 \mathrm{~cm}^{-1}$ in the spectrum of AS2-Water is slightly wider and shifted compared to that in the spectrum of AS1-Water at full CO saturation of the surface (Figure S4).
At a higher CO fractional coverage (Figure S5) of the surface, the saturation of the $\tilde{v}=2206 \mathrm{~cm}^{-1}$ band is reached, that is, the medium and soft Lewis acid sites are saturated. A semiquantitative data analysis of the $\mathrm{CO}$ adsorption by FTIR spectroscopy was performed by modelling the contributions of the strong Brønsted, Brønsted and Lewis acid sites using Gaussian functions. The areas of the observed peaks were quantified as the $\mathrm{CO}$ saturation coverage was reached and then normalised using the total mass of the sample. The obtained area per gram of sample $\left[\mathrm{m}^{2} \mathrm{~g}^{-1}\right]$ was then normalised by the BET specific surface area to give a value of the density of the acid sites in arbitrary units per square metre [a.u. $\mathrm{m}^{-2}$ ]. The results indicate noticeable differences between the samples with regard to the density of the acid sites (Table 1). Notably, two types of

\begin{tabular}{|c|c|c|c|c|}
\hline Sample & $\begin{array}{l}\text { Strong } \\
\text { Lewis }\end{array}$ & $\begin{array}{l}\text { Medium or soft } \\
\text { Lewis }\end{array}$ & $\begin{array}{l}\text { Strong } \\
\text { Brønsted }\end{array}$ & $\begin{array}{l}\text { Brønsted } \\
\mathrm{AlOH}\end{array}$ \\
\hline AS1 & 7 & 80 & 4 & 258 \\
\hline AS1-Water & 4 & 19 & 12 & 236 \\
\hline AS2 & 9 & 25 & 34 & 169 \\
\hline AS2-Water & 7 & 17 & 36 & 350 \\
\hline
\end{tabular}

Brønsted sites can be observed: a strong one, close to $\tilde{v}=$ $2175 \mathrm{~cm}^{-1}$ and a softer one, close to $\tilde{v}=2167 \mathrm{~cm}^{-1}$. However, only AS2-Water exhibits a detectable amount of very strong Brønsted sites. We used ${ }^{27} \mathrm{Al}$ MAS NMR spectroscopy to analyse the presence of $\mathrm{Al}^{\mathrm{V}}$ (sites located generally at the interface between alumina and silica or in aluminosilicate domains), which were not detected in AS1 and AS1-Water (Figure S6). AS1 only shows a low proportion of $\mathrm{Al}^{\mathrm{IV}}$ and is composed predominantly of $\mathrm{Al}^{\mathrm{Vl}}$. The other samples have in their volume a proportion of $30 / 70 \%$ of tetrahedral/octahedral Al, characteristic of $\gamma$-alumina (Table 2).

Before we acquired the EFTEM tilt series, a classical investigation of the global chemical composition of the grains was performed by using energy-dispersive X-ray (EDX) spectroscopy. This first step consisted of the study of the general composition of the samples by analysing several representative grains. The grains with a diameter below $\approx 20 \mathrm{~nm}$ consist only of alumina. This can be explained by the fact that alumina represents the major proportion in the mixture and by the relative heterogeneity between the components, below a specific grain size only a small proportion of grains contain silica in a non-negligible amount. Above this critical size, the grains have both components in various proportions. The obtained

\begin{tabular}{|lccc|}
\hline \multicolumn{4}{|l|}{ Table 2. Speciation of $\mathrm{Al}$ by using ${ }^{27} \mathrm{Al}$ MAS NMR spectroscopy. } \\
Sample & $\mathrm{Al}^{\mathrm{V}}[\%]$ & $\mathrm{Al}^{\mathrm{V}}[\%]$ & $\mathrm{Al}^{\mathrm{Vl}}[\%]$ \\
\hline AS1 & Trace & - & 100 \\
AS1-Water & 28 & - & 72 \\
AS2 & 23 & 7 & 70 \\
AS2-Water & 23 & 11 & 67 \\
\hline
\end{tabular}


EDX results on the majority of the studied grains (small and large) were in good agreement with the global composition obtained by using XRF spectroscopy. Among the analysed grains and because of the heterogeneity of the sample at the nanometre scale for the EFTEM tomography analyses, we selected grains that had a proportion of approximately $50 \%$ $\mathrm{Al}_{2} \mathrm{O}_{3}$ and $50 \% \mathrm{SiO}_{2}$ according to EDX quantification to maximise the SNR in both the chemical selective projections (Al and Si; Table S5).

\section{EFTEM tomography}

It is well known that the amorphous calcined silica-alumina complexes are heterogeneous materials at the nanometre scale, and this is generally independent of the synthesis method used. ${ }^{[17]}$ To confirm the protocol applied to extract the chemical signals of $\mathrm{Si}$ and $\mathrm{Al}$ in the energy-filtered images, several grains made exclusively with a single component, alumina or silica, similar in size and morphology with those that constitute the mixed grains, were analysed. In this way it was possible to isolate the pure energy electron loss spectroscopy (EELS) signal of Al and $\mathrm{Si}$ and to compute the EELS signals for the silica-alumina grains with their relative amount and compare them with those obtained from EDX spectroscopy. The EELS spectra were recorded using the same exposure time, dispersion and beam intensity. The assumption was made that the total amount of an aluminosilicate phase is negligible inside the sample, in agreement with the results obtained by using FTIR spectroscopy. The similarity between the corresponding calculated spectrum (blue) and the experimental spectrum for AS1-Water (magenta) can be observed in Figure 1. Only a small discrepancy can be observed between the spectra, probably because of a small difference between the thicknesses of the single-component grains and that of the AS1-Water grains. Additionally, the zero loss (ZL) and chemical projections of $\mathrm{Al}$ and $\mathrm{Si}$ at $0^{\circ}$ tilt angle extracted from the tilt series used for the volume reconstruction is shown in Figure 1. The reconstructed volumes were examined and visualised at different depths and orientations (Figure S7), and a different colour was assigned to each element: red for Al and green for Si.

The four silica-alumina samples were analysed by using EFTEM tomography. The distribution of the two individual components at the surface and a typical cross-section through the $3 \mathrm{D}$ corresponding model, parallel to the same view plane, are displayed in Figure 2. A simple analysis of these 3D representations shows that the initial samples AS1 and AS2 are quite heterogeneous. Silica generally constitutes the "core" of the sample; indeed, with a global proportion of $50 \%$, only and $30 \%$ of the total surface of the grain is made of silica. AS1Water and AS2-Water have a comparable proportion of the
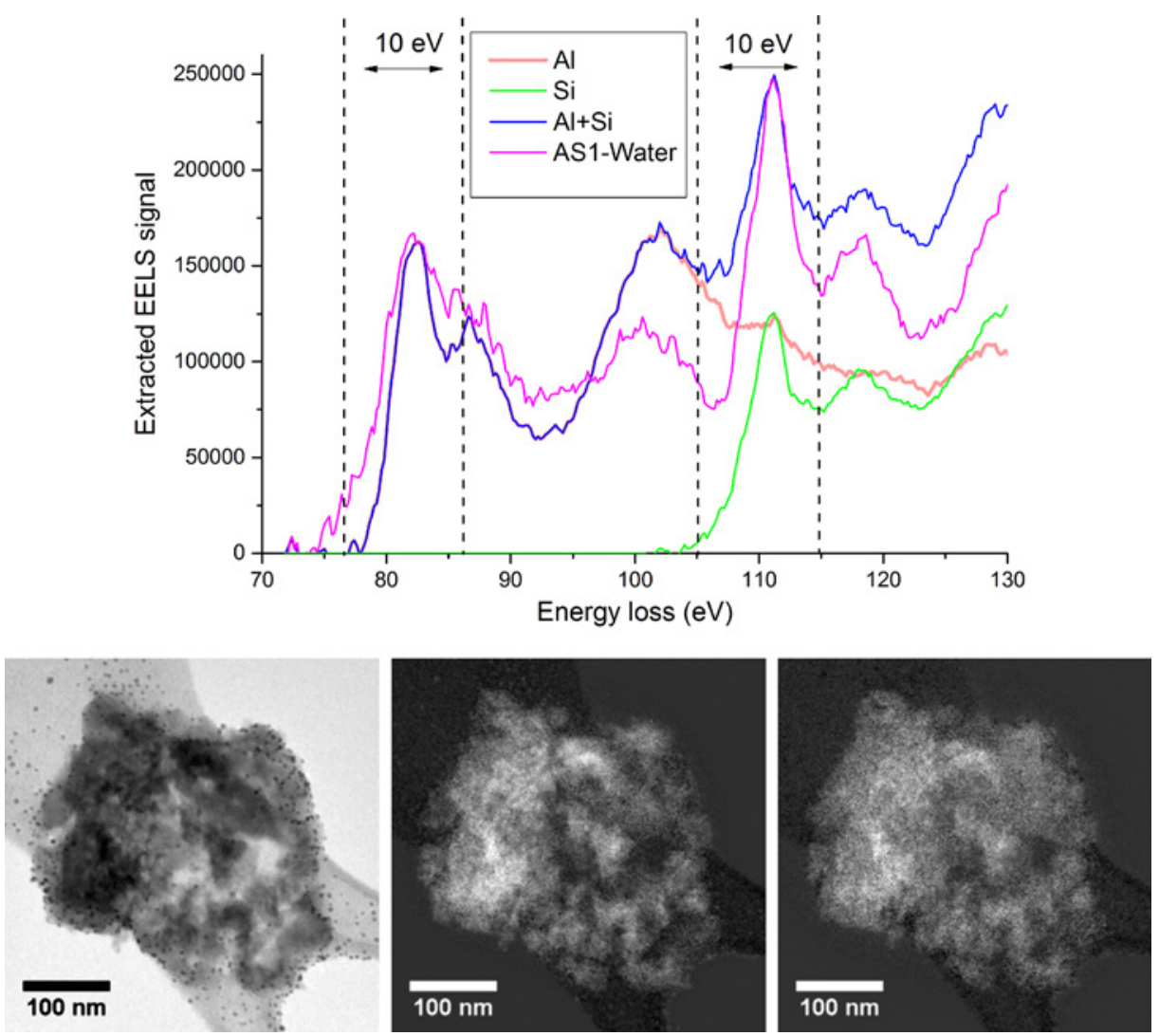

Figure 1. Top: The EELS signals of Al and of Si extracted from single-component alumina and silica grains, which have been used to calculate the signal provided by a mixed silica-alumina grain with a concentration of $50 \%$ alumina and $50 \%$ silica. Despite a small difference, the computed EELS signal (Al+Si in blue) and the EELS signal extracted from the sample AS1-Water are similar; the dashed lines represent the positions and the widths of the post-edge filtered images for $\mathrm{Al}$ and Si. Bottom: left) ZL projection at $0^{\circ}$ of AS1-Water, middle) the corresponding Al chemical projection and right) Si chemical projections. 

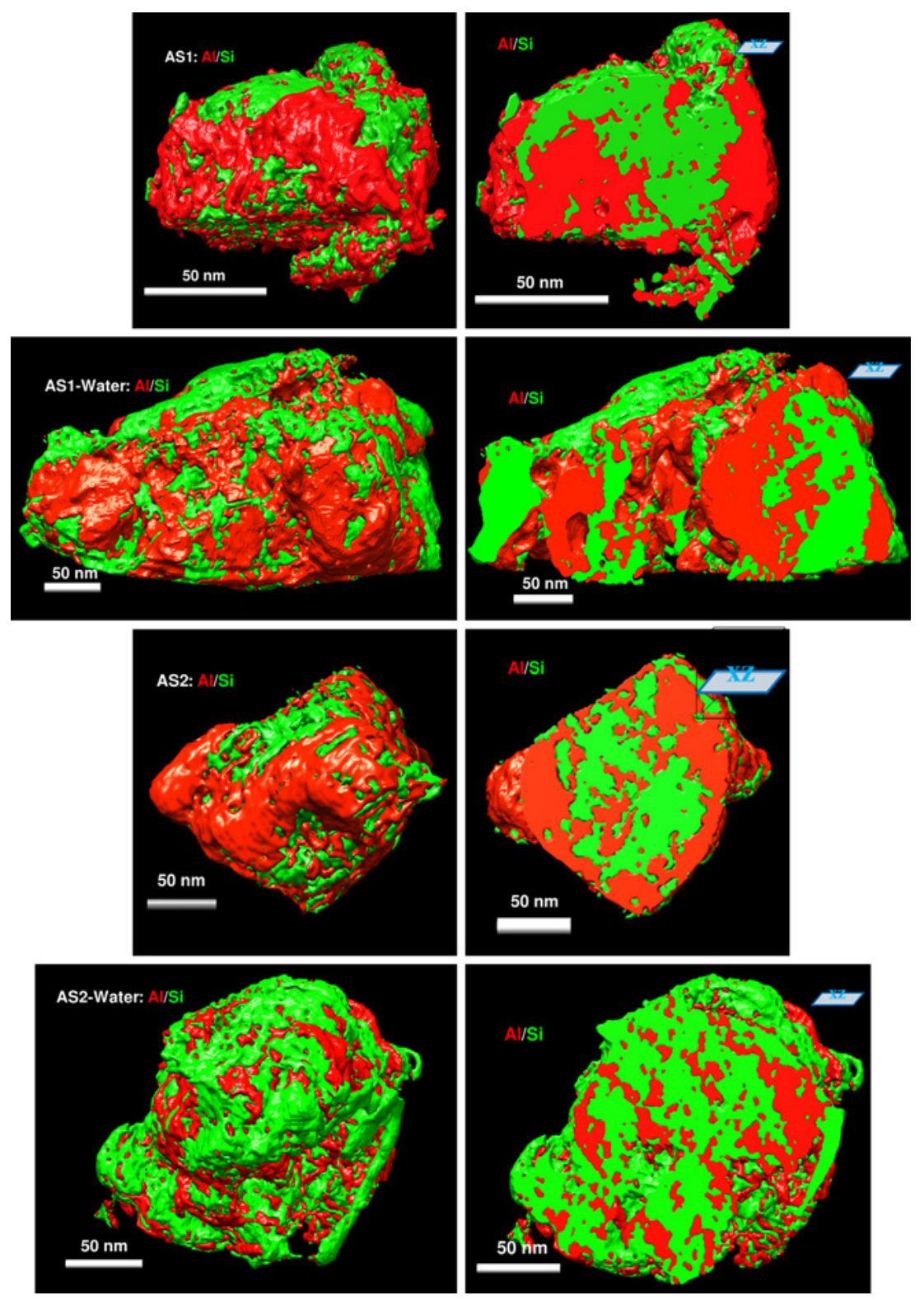

Figure 2. Left: 3D chemical surface models of some representative grains of the four analysed samples determined by using EFTEM tomography quantification. Right: typical slices parallel to the $x z$ plane through the $3 \mathrm{D}$ models, which shows the distribution of the components in the volume of the grains.

components at the surface of the grain, approximately $50 \%$ for both Si and Al (Table 3).

\begin{tabular}{|c|c|c|c|c|c|c|}
\hline \multirow[t]{2}{*}{ Sample } & \multirow{2}{*}{$\begin{array}{l}\text { Estimated } \\
\text { resolution } \\
{[\mathrm{nm}]}\end{array}$} & \multirow{2}{*}{$\begin{array}{l}\text { Specific surface } \\
\text { area } \\
{\left[\mathrm{m}^{2} \mathrm{~g}^{-1}\right]}\end{array}$} & \multicolumn{2}{|c|}{$\begin{array}{c}\text { Volume } \\
\text { proportion [\%] }\end{array}$} & \multicolumn{2}{|c|}{$\begin{array}{c}\text { Grain surface } \\
{[\%]}\end{array}$} \\
\hline & & & $\mathrm{SiO}_{2}$ & $\mathrm{Al}_{2} \mathrm{O}_{3}$ & $\mathrm{SiO}_{2}$ & $\mathrm{Al}_{2} \mathrm{O}_{3}$ \\
\hline AS1 & 4 & 79 & $51 \pm 3$ & $48 \pm 3$ & $31 \pm 3$ & $68 \pm 3$ \\
\hline AS1-Water & 12 & 33 & $54 \pm 3$ & $46 \pm 3$ & $49 \pm 1$ & $51 \pm 1$ \\
\hline AS2 & 7 & 30 & $50 \pm 2$ & $50 \pm 2$ & $34 \pm 3$ & $65 \pm 3$ \\
\hline AS2-Water & 7 & 38 & $54 \pm 3$ & $45 \pm 3$ & $51 \pm 1$ & $49 \pm 1$ \\
\hline
\end{tabular}

\section{Alumina-silica boundary line}

The acid properties needed for catalytic applications are provided by the aluminosilicate phases that result from an intimate mixing between silica and alumina. In this study, given the demonstrated heterogeneity of silica and alumina distributions, it was assumed that the aluminosilicate is only located where a physical mixing between alumina and silica particles takes place through atomic diffusion. Consequently, the active acid sites are expected to be located at the interface between the alumina and silica particles and reach the surface of the grains. The length of the silica-alumina interface at the surface of the individual grains can be defined as the "alumina-silica boundary line" at which the main catalytic activity can occur. This line is probably broadened by the atomic diffusion ${ }^{[16]}$ of $\mathrm{Al}$ 

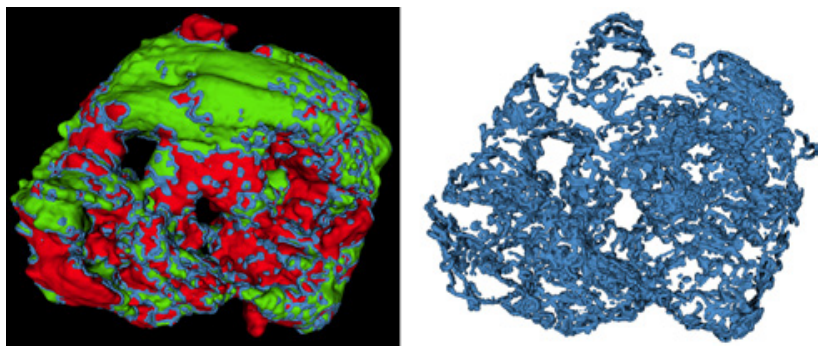

Figure 3. Representation of the catalytic alumina-silica boundary line (blue), alumina (red) and silica (green) for AS1-Water. The width of the line was expanded artificially to make it more visible.

and Si that probably leads to the appearance of a "mixture" band at the surface. The resolution of EFTEM tomography does not allow us to solve the width of this band directly so far. However, a representation of the alumina-silica boundary line distribution on the grain surface deduced for AS1-Water is shown in Figure 3. An estimate of the total length of this alumina-silica boundary line for the analysed grain was possible by selecting the pixels that separate silica and alumina at the surface of the grain and measuring the length of the curve they form. The length of the alumina-silica boundary line was normalised and expressed in $\mathrm{mg}^{-1}$ or in $\mathrm{mm}^{-1}$ (by dividing the length by the mass of the analysed grains respectively by the specific surface area calculated by using EFTEM tomography). A geometrical and chemically selective parameter was obtained, defined as the "specific boundary length" $\left[\mathrm{mm}^{-1}\right]$ (Table 4). The analysis of values from Table 4 leads us to conclude that after the thermal treatment even if an increase of the silica proportion at the surface of the samples was observed, the length of the alumina-silica boundary line decreases.

\section{Discussion}

The 3D analysis performed by using EFTEM tomography shows that the distribution of alumina and silica is rather heterogeneous in the initial samples before thermal treatment. In both samples, prepared by PSB and MM, the surface is covered preferentially with alumina, and silica remains in the core of the grains. The presence of large alumina domains on the samples was confirmed by XPS, NMR and FTIR spectroscopy. The micropores are provided mainly by silica and the mesopores by alumina, which seems less compacted and embeds the silica as a core. The same phenomenon was observed on titani-

\begin{tabular}{|c|c|c|}
\hline Sample & $\begin{array}{l}\text { Alumina-silica } \\
\text { boundary line length } \\
{\left[\mathrm{mg}^{-1}\right]}\end{array}$ & $\begin{array}{l}\text { Specific boundary } \\
\text { length } \\
{\left[\mathrm{m} \mathrm{m}^{-1}\right]}\end{array}$ \\
\hline AS1 & $390 \times 10^{8}$ & $4.94 \times 10^{8}$ \\
\hline AS1-Water & $177 \times 10^{8}$ & $5.36 \times 10^{8}$ \\
\hline AS2 & $335 \times 10^{8}$ & $11.55 \times 10^{8}$ \\
\hline AS2-Water & $170 \times 10^{8}$ & $4.47 \times 10^{8}$ \\
\hline
\end{tabular}

um oxide-alumina catalyst supports; if the proportion of titanium oxide was more than $30 \mathrm{wt} \%$ alumina embedded the titanium oxide. ${ }^{[34]}$

The PSB AS2 sample shows a better homogeneity than the MM AS1 sample, which is expected from the smaller size of the silica precursor used to prepare AS2 (silicic acid oligomers) than that of the precursor used for AS1 (silica colloidal particles). Surprisingly, AS1 shows an asymmetric AI2p XPS peak, which is compatible with an aluminosilicate phase and provides direct evidence for the intimate contact between silica and alumina at the surface of the grains. However, NMR spectroscopy confirms the heterogeneity of alumina and silica domains in the sample from the small amount of penta-coordinate $\mathrm{Al}^{\mathrm{V}}$ sites, which shows that the aluminosilicate phases represent a minor proportion of the total volume of the samples. As expected from samples obtained through simple mechanical preparation (MM), the AS2 sample exhibits stronger Brønsted acid sites than AS1 because the Si precursor is in a different state. Fresh silicic acid, supposed to contain only small silicic acid oligomers, is expected to produce far more reactive species if combined with $\mathrm{Al}$ than a colloidal suspension such as that used to prepare the first sample.

The thermal treatment of the samples is accompanied by a growth of the pore diameters and a decrease of the specific surface area, as observed by using $\mathrm{N}_{2}$ adsorption and confirmed by using EFTEM tomography. The porous volumes remain rather constant after the thermal treatment for both samples as the pore diameters increases slightly, which can be explained by a diminution of the small mesoporosity and probably also of the microporosity inside the grains. This is compatible with the generation of mobile $\mathrm{Si}$ species under steaming conditions, ${ }^{[11,12]}$ and the small mesoporosity originates essentially from silica-rich zones.

The most important effect of the thermal treatment is a redistribution of silica/alumina mobile species inside the grains, between the volume and the surface and at the surface of the grains. EFTEM tomography of the samples has shown an increase of the silica amount at the surface of the grains that reaches $50 \%$ of the total surface of the analysed grains. As the mobile Si species can be transported few tens of microns or more away, ${ }^{[13]}$ they can easily cross the distance of the EFTEM grains (a few hundred nanometres). From a more general point of view, all the obtained results show similar characteristics of the treated samples AS1-Water and AS2-Water in terms of the spatial distribution of silica and alumina in the grains. If we start from a more heterogeneous microstructure, the consequences of the redistribution of the components in the volume of the samples caused by the thermal treatment are more important for AS1 obtained through MM. However, the components redistribution was also observed for AS2.

The redistribution of silica at the surface induced by the thermal treatment implies a modification of the types and strength of acid sites as shown by using FTIR spectroscopy. These results show that for AS1 a large amount of the Brønsted acid sites are formed during the thermal treatment. From this, it can be concluded that the PSB method provides an ini- 
tial sample with more Brønsted acid sites compared to the MM method used to prepare AS1.

FTIR spectroscopy indicates a general enhancement of the Brønsted acid site density, and XPS analysis detects a reduction of the alumina and oxygen contents at the grain surface, which corresponds to a considerable modification of the surface chemistry. The decrease of the alumina proportion at the surface is in agreement with the reduction of the Lewis acid site density that is only provided by alumina. ${ }^{[23]}$ The oxygen density reduction at the surface, observed by using XPS, may be also related to a decrease of the amount of hydroxyl compounds, and can thus also contribute to the enhancement of the number of $\mathrm{Si}-\mathrm{OH}-\mathrm{Al}$ Brønsted acid sites observed by using FTIR spectroscopy after the thermal treatment. More specifically, for AS1 the thermal treatment reduces the surface density of strong Lewis acid sites significantly, whereas the strong Brønsted acid site density is enhanced. Notably, the small amount of silica observed on the surface of this sample can explain the difficulty to quantify the signal of the strong Brønsted sites properly. In the case of AS2, the Lewis and strong Brønsted site density remains almost constant after the thermal treatment, whereas the Brønsted $\mathrm{Al}-\mathrm{OH}$ sites density is enhanced clearly, which thus leads to a broadening of the distribution of the acidic forces. However, the two thermally treated samples AS1-Water and AS2-Water exhibit only small differences in the distribution of acidic forces that correspond to strong Brønsted sites.

The information obtained from electron microscopy studies is local and that obtained by using EFTEM tomography is not at the atomic level. More precisely, the $3 \mathrm{D}$ resolution in EFTEM tomography estimated for the analysed grains is $\approx 5 \mathrm{~nm}$. This explains why the specific surface areas of the analysed grains are approximately ten times lower that the values determined by using BET analysis. Even so, the results of EFTEM tomography are in agreement with those from XPS, except for AS2 and AS2-Water. This discrepancy lies in the fact that XPS suggests only minor modifications of the surface chemistry: no substantial changes in the $O$ content, whereas the change of the silica amount at the surface of the grains is observed in the spectra. However, a clear redistribution of the silica between the grain bulk and its surface was observed by using EFTEM tomography, with which the contribution of silica increases from 34 to $50 \%$ of the total surface. This is substantiated by using NMR spectroscopy, which shows a slight increase of the proportion of penta-coordinated $\mathrm{Al}^{\mathrm{V}}$ sites that grows from 7 to $11 \%$, and by using FTIR spectroscopy, which shows the formation of strong Brønsted $\mathrm{Si}-\mathrm{OH}-\mathrm{Al}$ acid sites at $\mathrm{CO}$ saturation.

The thermal treatment also influences the length of the alumina-silica boundary line estimated by using EFTEM tomography. A decrease of the mass-normalised active alumina-silica boundary line is observed after the thermal treatment. This result can be explained by the strong reduction of the specific surface area, confirmed by BET measurements, which can also be observed by using EFTEM tomography. If the values are normalised by the surface area of the grains, the variation of the alumina-silica boundary line lengths is not correlated with the $\mathrm{CO}$ adsorption measurements of the strong Brønsted acid site density. The explanation is that EFTEM does not reach the atomic resolution that is mandatory to quantify the width of the alumina-silica boundary line precisely and, consequently, the real density of the strong Brønsted acid sites. The thermal treatment enhances the diffusion at the interface between the alumina and silica individual domains and may increase the thickness of the alumina-silica boundary line, which explains the increase of the density of the strong Brønsted acid sites observed by using FTIR spectroscopy, even if the length of the line seems to diminish. In other words, steaming may not only change the size and arrangement of the silica- and aluminarich zones, it may also create molecular-sized silica islands on alumina-rich zones to generate Brønsted acid sites that cannot be imaged by using EFTEM.

Indeed, a one-to-one correlation between the length of the alumina-silica boundary line and the density of the strong Brønsted acid sites is difficult to obtain by studying real samples used in industrial application daily. This is because a real bridge between the nano and meso scale is hard to obtain; this may because an action at the mesoscale cannot really influence the nanoscale. Nevertheless, this bridge could be revealed by analysing a "school" sample specially built for highresolution analysis by orientating the samples well and measuring the width of the atomic diffusion between the individual silica and alumina grains and so the thickness of the aluminasilica boundary line.

A comparison between the two types of materials gives a first insight into each preparation technique. If we start from heterogeneous materials, thermal treatment can improve the homogeneity of the mixture but its effect is limited by the size of the individual grains at a nanometre scale. As AS2 is basically at the limit of the homogeneity criterion, the thermal treatment has a limited influence at the nanometre scale of the individual silica and alumina domains. One solution can be the employment of a longer thermal treatment time or of a higher temperature, if the induced specific surface area loss is acceptable for the considered application. Nevertheless, the strong impact of the thermal treatment at the meso scale cannot completely equalise the synthesis method that provides the starting homogeneity between the nanometric individual grains of silica and alumina.

The same analytical methodology could be, in principle, applied to the analysis of other types of materials, such as nanomaterials used for fuel cell applications or in electrocatalysis applications, in which an interface between two different compounds that reaches the surface of the grain is generally present.

\section{Conclusion}

Electron tomography in general and in particular energy-filtered transmission electron microscopy (EFTEM) tomography provides a valuable $3 \mathrm{D}$ characterisation of a catalyst support and active phase. We used mechanical mixing and the precipitation of silica on boehmite (PSB) to prepare two amorphous silica-alumina catalyst supports, which exhibit a heterogeneous distribution of the components. The quantitative parameters 
extracted by using EFTEM tomography show that the raw materials are a heterogeneous mixture of silica and alumina with a predominance of the alumina compound on the surface. Based on this $3 \mathrm{D}$ chemical analysis, it can be concluded that the PSB method provides grains with a better homogeneity of the silica and alumina compounds at the nanometre scale than mechanical mixing. The PSB sample is thus characterised by a higher density of Brønsted $\mathrm{Si}-\mathrm{OH}-\mathrm{Al}$ acid sites and a higher proportion of $\mathrm{Al}^{\mathrm{V}}$, characteristic of aluminosilicate domains, as deduced by using FTIR and NMR spectroscopy.

A thermal treatment performed in a humid atmosphere induces the redistribution of the compounds in the volume and onto the surface of the materials, the reduction of the specific surface area and a modification of the type and the density of the acidic sites at the surface of the grains. More precisely, it leads to an increase of the silica amount at the surface of the grains that covers almost $50 \%$ of the total surface for both analysed samples. For the PSB sample, the effect of the treatment on the components redistribution is limited because of a more homogenous initial distribution, characterised by alumina and silica domains with a nanometre size. In such a case, the thermal treatment did not improve the mixing between these individual domains substantially but it stimulates the diffusion of $\mathrm{Si}$ and $\mathrm{Al}$ atoms at the interface between them, which thus increases the density of the acid sites on the surface. The final result is that the two thermally treated samples exhibit only small differences in the density of acidic forces that correspond to the Brønsted acid sites.

The length of the curve created by crossing the aluminasilica interface and the surface of the grains was measured and compared to the density of acid sites measured by using FTIR spectroscopy after CO adsorption. It was observed that, though the length of this line on the surface decreases, the density of the acid site increases because of the thermal diffusion of the silica and alumina at the interface between the individual domains.

\section{Experimental Section}

\section{Sample preparation}

Two initial samples were synthesised by two methods. AS1 was obtained by MM from boehmite powder and silica powder. AS2 was synthesised by the PSB method from a mixture of a boehmite powder with a colloidal solution of silicic acid. The samples were calcined between 550 and $600^{\circ} \mathrm{C}$ in a dry (AS1, AS2) or humid atmosphere (AS1-Water, AS2-Water). As the silica precursor in AS2 (silicic acid oligomers) is smaller than that in AS1 (silica colloidal suspension), silica is expected to be dispersed more homogeneously on AS2 than on AS1.

AS1 was prepared from boehmite (150 g; SASOL, PURAL ${ }^{\circledR}$ SB3) mixed with nitric acid solution $(150 \mathrm{~g}, 2.1 \mathrm{wt} \%)$. This mixture was added to a suspension ( $1 \mathrm{~L}$ ) of silica (NYACOL, Nyasil $5{ }^{\circledR}$ ) in water $(5.5 \mathrm{wt} \%)$ and heated at $80^{\circ} \mathrm{C}$ for $1.5 \mathrm{~h}$ with stirring. The alumina and silica precursors were weighed to obtain a $70 \mathrm{wt} \%$ alumina/30 wt $\%$ silica ratio. The suspension was fil- tered, and the resulting solid was extruded through a cylindrical hole with a $1.4 \mathrm{~mm}$ diameter. The extrudates were dried at $120^{\circ} \mathrm{C}$ during $24 \mathrm{~h}$ and then calcined at $550^{\circ} \mathrm{C}$ for $4 \mathrm{~h}$ with a dry air flow. AS1-Water was obtained after the calcination of AS1 in the presence of water vapour at $750^{\circ} \mathrm{C}$.

AS2 was obtained by using the method described by Euzen et al (example 5). ${ }^{[41]}$ Silicic acid was obtained by the exchange of a silicate solution (UNIVAR, Collonges type) on a cationic exchange resin (BAYER, Lewatit MonoPlus S100) and filtered on a membrane (MILLIPORE NMWL 30000). Boehmite (SASOL, PURAL ${ }^{\circledR}$ SB3) and silicic acid solution were weighed to obtain a $70 \mathrm{wt} \%$ alumina $/ 30 \mathrm{wt} \%$ silica ratio. The precursors were mixed under vigorous stirring with $8 \mathrm{wt} \%$ nitric acid relative to the aluminosilicate solid. The amount of nitric acid was set accurately to a value that ensures the complete peptisation of the Al precursor, which has no significant influence on its condensation and on the polymerisation of aluminosilicate species. The resulting suspension was dried by using an atomiser in a conventional manner. The as-prepared powder was extruded through a cylindrical hole with a $1.4 \mathrm{~mm}$ diameter. The extrudates were dried at $150^{\circ} \mathrm{C}$ in a ventilated oven, and then calcined at $550^{\circ} \mathrm{C}$ using a dry air flow. AS2-Water was obtained after the calcination of AS2 in the presence of water vapour at $750^{\circ} \mathrm{C}$.

\section{Bulk and surface traditional characterisation}

The bulk Al and Si compositions of the samples were obtained by using wavelength-dispersive XRF spectroscopy. Samples were crushed in a mortar, diluted by $\approx 50$ times in a lithium, borate and phosphate glass and moulded at high temperature to form glass pearls. The quantitative analysis was performed by using a calibration curve obtained from reference pearls that contained a weighed amount of alumina and silica. The volume fractions were extracted from the XRF composition by assuming that the samples are composed only of non-porous clusters of only pure alumina and silica with a density of 3.97 and $2.533 \mathrm{~g} \mathrm{~cm}^{-3}$, respectively.

The surface composition was obtained by using XPS. Samples were crushed in a mortar and put into an In foil. The monochromated AlK $\mathrm{A}_{\alpha}$ source of a KRATOS Axis Ultra was used at $10 \mathrm{kV}$ and $15 \mathrm{~mA}$. Survey spectra were acquired with $160 \mathrm{eV}$ pass energy, and individual peaks (O $1 \mathrm{~s}, \mathrm{Al} 2 \mathrm{p}$ and $\mathrm{Si} 2 \mathrm{p})$ were measured with a pass energy of 5, 10 and $40 \mathrm{eV}$. An electron flood gun was used to limit charging phenomena by keeping the carbon peak from contamination at $B E=284.6 \mathrm{eV}$. Each sample was prepared and measured twice and showed good reproducibility. Peak decomposition was performed with the Kratos Vision 2 software based on non-linear least-squares fitting of the weighted sum of Lorentzian and Gaussian components super-posed to a background calculated by the model of Shirley and Sherwood. ${ }^{[42]}$ The precision of the binding energy is approximately $\pm 0.1 \mathrm{eV}$. Surface concentrations were obtained after a careful calibration of the transmission factor of the spectrometer. 
$\mathrm{N}_{2}$ adsorption-desorption isotherms were recorded at $77 \mathrm{~K}$ by using a Micromeretics ASAP 2000 apparatus after the pre-treatment of samples under vacuum for $4 \mathrm{~h}$ at $450^{\circ} \mathrm{C}$. Surface area values were obtained by using the BET model. Porous diameters were calculated by using the Barret-Joyner-Halenda method with the adsorption branch of the isotherm. ${ }^{[43]}$

CO adsorption was studied by using FTIR spectroscopy by using a Thermo Nicolet Nexus spectrometer with a resolution of $4 \mathrm{~cm}^{-1}$. Samples were crushed in a mortar and pressed to form a self-supporting cylindrical pellet. They were pre-treated at $350^{\circ} \mathrm{C}$ for $10 \mathrm{~h}$ under secondary vacuum to remove adsorbed water. Several controlled pulses of $\mathrm{CO}$ at $-196^{\circ} \mathrm{C}$ were used on an adequate pellet thickness to extract the concentration of $\mathrm{CO}$ adsorbed on the acid sites quantitatively from the Beer-Lambert law. The absorbance $A$, the pellet compacted thickness $I$ and the concentration of sites $c$ are related by $A=$ $\varepsilon l c$, in which $\varepsilon$ is the molar extinction coefficient, characteristic of the adsorbed species. Unfortunately, the lack of reference on alumina-silica samples with known concentration of sites prevents $\varepsilon$ measurement and hence an absolute determination of $c$. Anyway, it is possible to make a comparison between samples in absorbance units if we assume that: (a) All the samples have the same cylindrical section, (b) the compactness of the pellets is the same, (c) the samples have a similar bulk composition and (d) the same adsorption sites are present in all samples. The number of sites $N_{\mathrm{m}}$ [a.u./g] is obtained by dividing $A$ by the mass of the pellet. It is then possible to compare the number of sites per surface unit $N_{\mathrm{s}}$ by dividing $N_{\mathrm{m}}$ by the specific surface area (BET), providing that nitrogen and $\mathrm{CO}$ access the same surface, which seems a valid assumption for a non-microporous sample. To enhance the spectral resolution and to be able to discriminate the $\mathrm{AlOH}$ and the strong Brønsted acid sites, we applied a Fourier self-deconvolution (FSD) treatment between $\tilde{v}=2240$ and $2080 \mathrm{~cm}^{-1}$. The FSD method enhances the possibility to discriminate individual IR bands without the need to increase the instrumental resolution. Two parameters are important to perform this specific signal treatment of an IR spectrum. The first one is the adjustment of the width $X$ of the apodisation function. The second one is the enhancement factor $k$ that is the ratio of the band width before FSD to that after FSD. To perform the FSD-IR treatment, it is critical to start with a good SNR in the original spectrum. In this present work, FSD was optimised with an $X$ of $15.7 \mathrm{~cm}$ and a $k$ of 2.5. For further details of the FSD-IR, we refer readers to the guide of the method by Griffiths and Pariente. ${ }^{[44]}$

${ }^{27} \mathrm{AI}$ MAS NMR spectroscopy was performed by using a Bruker Avance 400 spectrometer with a rotation frequency of $12 \mathrm{kHz}$. Chemical shifts are referenced to a liquid solution of $\mathrm{Al}\left(\mathrm{NO}_{3}\right)_{3}$ set at $\delta=0 \mathrm{ppm}$. As the ${ }^{27} \mathrm{Al}$ nucleus is quadrupolar, the attribution of coordination state from the chemical shifts is not straightforward. In particular, some penta-coordinated Al has a similar chemical shift that causes the distortion of tetra-coordinated ones. Anyway, MAS NMR spectroscopy used in stable conditions of high static magnetic field, high spinning frequency and high water partial pressure ${ }^{[45]}$ can provide a good estimation of the populations of the coordination states. Chemical shifts are attributed to: octahedral $\mathrm{Al}\left(\mathrm{Al}^{\mathrm{Vl}}\right)$ in the $\delta=-20$ $10 \mathrm{ppm}$ range, penta-coordinated $\mathrm{Al}\left(\mathrm{Al}^{\mathrm{V}}\right)$ in the $\delta=20$ $40 \mathrm{ppm}$ range and tetrahedral $\mathrm{Al}\left(\mathrm{Al}^{\mathrm{IV}}\right)$ in the $\delta=50-70 \mathrm{ppm}$ range. ${ }^{[16]}$ The proportion of each kind of coordination for $\mathrm{Al}$ was obtained by the integration of the NMR signal intensities between the aforementioned chemical shifts. For interpretation, it is known that transition alumina contains both $\mathrm{Al}^{\mathrm{IV}}$ and $\mathrm{Al}^{\mathrm{VI}}$ in a roughly $30-70 \%$ proportion for $\gamma$-alumina. ${ }^{[46]} \mathrm{Al}^{\mathrm{V}}$ is located at the interface between alumina and silica or in aluminosilicate domains. ${ }^{[4]]}$

\section{Analytical EFTEM tomography}

EFTEM tomography analyses were performed by using a JEOL $2100 \mathrm{~F}$ transmission electron microscope equipped with a postcolumn GATAN Tridiem energy filter. First, the samples were crushed, dispersed in ethanol and ultrasonicated for $5 \mathrm{~min}$. A drop of the resulting solution was deposited on a microscopy holey carbon grid and dried for $10 \mathrm{~min}$ under a heating lamp. A drop of a suspension of colloidal Au particles was laid as fiducial markers for the alignment of the tilt series on the grid and dried once more. The tilt series was recorded automatically by using the GATAN tomography software. At each tilt angle, seven filtered images $10 \mathrm{eV}$ wide were recorded: one centred at the ZL peak used to obtain a density map, three centred at 59, 70 and $81 \mathrm{eV}$ used to isolate the signal that corresponds to the $L_{23}$ edge of $A l$, two centred at 99 and $110 \mathrm{eV}$ used to extract the signal that corresponds to the $L_{23}$ edge of Si and finally a last image at the ZL peak to quantify the drift of the sample during the acquisition of the filtered images at the same tilt angle. The recording time was $5 \mathrm{~s}$ for the filtered images acquired on the $\mathrm{Al}_{23}$ edge, $13 \mathrm{~s}$ on the $\mathrm{Si}_{23}$ edge and $0.2 \mathrm{~s}$ on the ZL. The acquisition parameters were chosen to maximise the SNR and to minimise the contribution of the plasmon peak on the Al signal. The tilt series was recorded in an angular span from -71 to $+71^{\circ}$ with an increment of $4^{\circ}$ in a Saxton scheme ${ }^{[48]}$ during a total recording duration of $2 \mathrm{~h}$, which resulted in 55 images of $512 \times 512$ pixels with a pixel size of approximately 0.34 (AS1), 0.84 (AS1-Water), 0.56 (AS2) and $0.56 \mathrm{~nm}$ (AS2-Water). The electron dose during the experiments was $\approx 10^{8}$ electrons $\mathrm{s}^{-1} \mathrm{~nm}^{-2}$, and no visible irradiation damage was observed at the end of the acquisition process. This allows us to conclude that for such a specimen, the radiation damage does not limit the resolution in the analytical tomographic mode.

After the tilt series acquisition, the first step concerns the validation of the main requirement of the EFTEM tomography: $:^{[32]}$ the image intensity in the $2 \mathrm{D}$ chemical maps used to reconstruct the chemical volume should be proportional to the thickness-integrated number of atoms of the chosen element. This requires minor contributions of the multiple inelastic scatterings and of the elastic scattering, as well as a proper background approximation and extraction of chemical signal recorded in the energy-filtered images. More details on the validation protocol are given in the Supporting Information. 
A rigorous alignment was applied between the filtered images recorded at the same tilt angle. The chemical-sensitive projections of Al were computed by employing the three-windows method and that of $\mathrm{Si}$ by employing the R-ratio method ${ }^{[32]}$ (Figure 1). The alignment of the chemical tilt series was performed by employing the IMOD software ${ }^{[49]}$ using as a reference the fine alignment done on the $Z L$ tilt series in which the Au $5 \mathrm{~nm}$ calibrated particles were used as fiducial markers. Chemical volume computations were performed by employing 15 iterations of the algebraic reconstruction technique algorithm $(A R T)^{[50]}$ implemented in the TOMOJ/EFTETJ software. ${ }^{[51]}$ Finally, the $3 \mathrm{D}$ visualisation, surface rendering and quantification were performed by combining different tools implemented in the ImageJ software, ${ }^{[52]} 3 \mathrm{D}$ Slicer ${ }^{[53]}$ and Chimera. ${ }^{[54]}$ The employed EFTEM tomography methodology has been described in detail in Ref. [32] and reproduced in this work.

\section{Acknowledgments}

The authors express their gratitude to Prof. Krijn P. de Jong, Dr. Cuong Pham-Huu, Prof. Claude Esnouf and Dr. Christophe Pichon for helpful discussions during the course of these experiments. Philippe Lecour is warmly acknowledged for the XPS measurements and Anne-Lise Taleb for EFTEM measurements. We are also grateful to the French Ministry of Higher Education and Research, Conventions Industrielles de Formation par la REcherche (CIFRE) and IFP Énergies Nouvelles for funding the PhD grant.

Keywords: acidity $\cdot$ aluminum $\cdot$ scanning probe microscopy silicon · supported catalysts

[1] A. Corma, Chem. Rev. 1995, 95, 559.

[2] T. K. Phung, L. Proietti Hernández, A. Lagazzo, G. Busca, Appl. Catal. A 2015, 493, 77.

[3] T. K. Phung, G. Busca, Catal. Commun. 2015, 68, 110.

[4] A. Chaumonnot in Catalysis by Transition Metal Sulphides (Eds.: P. Raybaud, H. Toulhoat), Editions Technip, Paris, 2013, p. 225.

[5] G. Busca, Chem. Rev. 2007, 107, 5366.

[6] C. Marcilly, Acido-Basic Catalysis, Vol. 2, Editions Technip, Paris, 2005.

[7] M. Girgis, P. Tsao, Ind. Eng. Chem. Res. 1996, 35, 386.

[8] A. M. Venezia, V. La Parola, B. Pawelec, J. L. G. Fierro, Appl. Catal. A 2004, $264,43$.

[9] W. P. Schlaffer, C. Z. Morgan, J. N. Wilson, J. Phys. Chem. 1957, 61, 714

[10] U. A. Sedran, N. S. Figoli, React. Kinet. Catal. Lett. 1989, 39, 363.

[11] S. Sato, R. Takahashi, T. Sodesawa, C. Kobayashi, A. Miura, K. Ogura, Phys. Chem. Chem. Phys. 2001, 3, 885.

[12] M. Caillot, A. Chaumonnot, M. Digne, J. Van Bokhoven, ChemCatChem 2014, 6, 2976.

[13] X. Liu, J. Phys. Chem. B 1999, 103, 2647.

[14] C. Chizallet, P. Raybaud, ChemPhysChem 2010, 11, 105.

[15] D. G. Poduval, J. A. R. van Veen, M. S. Rigutto, E. J. M. Hensen, Chem. Commun. 2010, 46, 3466.

[16] E. J. M. Hensen, D. G. Poduval, P. C. M. M. Magusin, A. E. Coumans, J. A. R. van Veen, J. Catal. 2010, 269, 201.

[17] C. Sârbu, B. Delmon, Appl. Catal. A 1999, 185, 85.

[18] I. Pieta, M. Ishaq, R. Wells, J. Erson, Appl. Catal. A 2010, 390, 127.

[19] P. Raybaud in Catalysis by Transition Metal Sulphides (Eds.: P. Raybaud, H. Toulhoat), Editions Technip, Paris, 2013, p. 387.
[20] R. Takahashi, S. Sato, T. Sodesawa, M. Yabuki, J. Catal. 2001, 200, 197.

[21] F. Leydier, C. Chizallet, A. Chaumonnot, M. Digne, E. Soyer, A. A. Quoineaud, D. Costa, P. Raybaud, J. Catal. 2011, 284, 215

[22] M. Caillot, A. Chaumonnot, M. Digne, J. A. van Bokhoven, J. Catal. 2014, $316,47$.

[23] W. Daniell, U. Schubert, R. Glöckler, A. Meyer, K. Noweck, H. Knözinger, Appl. Catal. A 2000, 196, 247.

[24] A. Bonduelle-Skrzypczak, C. Legens in Catalysis by Transition Metal Sulphides (Eds.: P. Raybaud, H. Toulhoat), Editions Technip, Paris, 2013 p. 254.

[25] P. Bayle-Guillemaud, A. Barbier, C. Mocuta, Ultramicroscopy 2001, 88, 99.

[26] P. J. Thomas, P. A. Midley, Top. Catal. 2002, 21, 109.

[27] H. Friedrich, P. E. de Jongh, A. J. Verkleij, K. P. de Jong, Chem. Rev. 2009, 109, 1613.

[28] G. Prieto, J. Zečević, H. Friedrich, K. P. de Jong, P. E. de Jongh, Nat. Mater. 2012, 12, 34.

[29] G. Möbus, R. C. Doole, B. J. Inkson, Ultramicroscopy 2003, 96, 433.

[30] P. A. Midgley, M. Weyland, Ultramicroscopy 2003, 96, 413.

[31] M. A. Aronova, Y. C. Kim, N. B. Pivovarova, S. B. Andrews, R. D. Leapman, Ultramicroscopy 2009, 109, 201.

[32] L. Roiban, L. Sorbier, C. Pichon, P. Bayle-Guillemaud, J. Werckmann, M. Drillon, O. Ersen, Microsc. Microanal. 2012, 18, 1118.

[33] I. Florea, O. Ersen, C. Hirlimann, L. Roiban, A. Deneuve, M. Houllé, I. Janowska, P. Nguyen, C. Pham, C. Pham-Huu, Nanoscale 2010, 2, 2668.

[34] L. Roiban, L. Sorbier, C. Hirlimann, O. Ersen, ChemCatChem 2016, 8, $1651-1657$.

[35] M. Digne, P. Sautet, P. Raybaud, P. Euzen, P. H. Toulhoat, J. Catal. 2004, 226, 54.

[36] G. Crépeau, V. Montouillout, A. Vimont, L. Mariey, T. Cseri, F. Maugé, J. Phys. Chem. B 2006, 110, 15172.

[37] O. Cairon, Phys. Chem. Chem. Phys. 2010, 12, 6333.

[38] C. Morterra, G. Magnacca, Catal. Today 1996, 27, 497.

[39] J. N. Kondo, R. Nishitani, E. Yoda, T. Yokoi, T. Tatsumia, K. A. Domenc, Phys. Chem. Chem. Phys. 2010, 12, 11576.

[40] M. Caillot, A. Chaumonnot, M. Digne, J. A. Van Bokhoven, ChemCatChem 2013, 5, 3644.

[41] P. Euzen, C. Bobin, M. Roy-Auberger, E. Benazzi, P. Bourges, C. Gueret, Patent, 2002, N ${ }^{\circ}$ FR 2846 574, B1.

[42] P. Sherwood in Practical Surface Analysis-Auger and X-ray Photoelectron Spectroscopy (Eds.: D. Briggs, M. Seah) Wiley, New York, 1990, p. 581.

[43] E. P. Barrett, L. G. Joyner, P. P. Halenda, J. Am. Chem. Soc. 1951, 73, 373.

[44] P. Griffiths, G. Pariente, TrAC Trends Anal. Chem. 1986, 5, 209.

[45] G. Engelhardt, D. Michel, High Resolution Solid State NMR of Silicates and Zeolites, Wiley, New York, 1987, p. 137.

[46] M. Williams, B. Fonfé, C. Sievers, A. Abraham, J. van Bokhoven, A. Jentys, J. van Veen, J. Lercher, J. Catal. 2007, 251, 485.

[47] B. de Witte, P. Grobet, J. Uytterhoeven, J. Phys. Chem. 1995, 99, 6961.

[48] W. Saxton, W. Baumeister, M. Hahn, Ultramicroscopy 1984, 13, 57.

[49] J. R. Kremer, D. N. Mastronarde, J. R. McIntosh, J. Struct. Biol. 1996, 116, 71.

[50] R. Gordon, R. Bender, G. T. Herman, J. Theor. Biol. 1970, 29, 471.

[51] C. Messaoudil, T. Boudier, C. O. Sanchez Sorzano, S. Marco, BMC Bioinformatics 2007, 8, 288.

[52] ImageJ, URL: http://imagej.nih.gov/ij/.

[53] A. Fedorov, R. Beichel, J. Kalpathy-Cramer, J. Finet, J. C. Fillion-Robin, S. Pujol, C. Bauer, D. Jennings, F. Fennessy, M. Sonka, J. Buatti, S. R. Aylward, J. V. Miller, S. Pieper, R. Kikinis, Magn. Reson. Imaging 2012, 30 1323, URL: http://www.slicer.org/.

[54] E. F. Pettersen, T. D. Goddard, C. C. Huang, G. S. Couch, D. M. Greenblatt, E. C. Meng, T. E. Ferrin, J. Comput. Chem. 2004, 25, 1605-1612, URL: https://www.cgl.ucsf.edu. 